\title{
Smart Parking Service based on Wireless Sensor Networks
}

\author{
Jihoon Yang, Jorge Portilla and Teresa Riesgo
}

\begin{abstract}
In this paper, we present the design and implementation of a prototype system of Smart Parking Services based on Wireless Sensor Networks (WSNs) that allows vehicle drivers to effectively find the free parking places. The proposed scheme consists of wireless sensor networks, embedded web-server, central web-server and mobile phone application. In the system, low-cost wireless sensors networks modules are deployed into each parking slot equipped with one sensor node. The state of the parking slot is detected by sensor node and is reported periodically to embedded web-server via the deployed wireless sensor networks. This information is sent to central web-server using Wi-Fi networks in real-time, and also the vehicle driver can find vacant parking lots using standard mobile devices.
\end{abstract}

\section{INTRODUCTION}

Wireless sensor network mote [1] is a tiny device which usually consists of a low cost-sensor module, a microprocessor module and a communication module, and provides a powerful consortium of distributed sensing, computing and communication. These modules can rapidly and easily be deployed to collect, process, and transmit information. The wireless sensor networks can be applied to many field areas [23] such as environmental monitoring [4], surveillance [5], smart home [6], agricultural [7], child education [8], emergency medical care with mobile device [9], etc.

In metropolitan areas, most vehicle drivers have the daily concern of finding a vacant parking space especially during the rush hours. It is time-consuming and it is leading to more traffic congestion, air pollution and driver frustration. A recent report [10] presents that the traffic congestion is generated by vehicles searching for parking spaces takes up to $45 \%$ of the total traffic. So, many parking management systems have been deployed in order to reduce such traffic congestion and improve the convenience for vehicle drivers. A few systems focused on the applications of vehicle parking system using video camera sensor technologies [11], [12] to collect the information in vehicle parking field. However, a video camera sensor is expensive, and can generate a large amount of data that can be difficult to transmit in wireless network. In recent years, wireless sensor networks technologies [13], [14] [16 21] have a great potential method for providing a low-cost solution in order to implement vehicle-parking service with respect to some reasons as following: easy deployment in existing parking lots without having to install new components (e.g. expensive line or cabling), and the flexibility to connect with sophisticated but cheap sensors for accurately keeping track of vehicles. Moreover, parking information can be gathered by each node and can be collaboratively processed to evaluate other meaningful metrics such as parking times, billing and payment, etc., to improve the benefit of vehicle drivers and managers of parking places.

This paper introduces a smart parking service based on wireless sensor networks and mobile phone application for vehicle drivers. We have designed and implemented a prototype system of smart parking services that allows vehicle drivers to effectively find the vacant parking spaces, both in outdoors and indoors environments. The proposed smart parking system consists of wireless sensor networks, embedded web-server, central web-server and mobile phone application as Android and iPhone. In this system, low-cost wireless sensors network modules are deployed into each parking slot equipped with one sensor node. The state of the parking slot is detected by sensor node and is reported periodically to the embedded web-server via the deployed wireless sensor network. And this information is sent to central web-server using WiFi networks in real-time, and the vehicle driver can also find vacant parking lots using a mobile phone or a tablet.

This paper is organized as follows. The related work in literature is presented in Section II. The architecture and operations of the proposed smart parking service are described in Section III. In Section IV, the prototype implementation of the smart parking systems are introduced. Finally, we conclude this paper in Section $\mathrm{V}$.

\section{RELATED WORK}

Some related works for the parking space management system based on wireless sensor networks are reviewed in this section.

SiPark [13] has developed solutions for efficient parking space management, including: intelligent parking guidance systems, convenient pay-and-display machines, and modern car park technology. Ultrasound sensors are used for detecting the presence or absence of a vehicle, and are connected using a network to distribute power and transmit sensing reports in SiPark systems. Tang et al. [14] developed an intelligent car parking system using wireless sensor networks where each parking space is equipped with one sensor to detect its availability. The implemented prototype model is based on Crossbow mote products [15] and the extended Crossbow 
XMesh network architecture. Caliskan et al. [16] proposed a parking place availability system using vehicular ad hoc networks (VANET) based on Wireless-LAN IEEE 208.11 to broadcast these reports as raw text packets. Benson et al. [17] presented car-park management using Wireless Sensor Networks based on their DSYS25z sensing nodes on Tiny OS 1.1.7. Boda et al. [18] presented the design and implementation considerations for a wireless sensor network that can track available parking spaces in public parking areas in real time and communicate the information to the users. They proposed a detection scheme using magnetometer signature measurements and they implemented it based on Mica2 Motes. $\mathrm{Lu}$ et al [19] introduced a new Smart PARKing (SPARK) scheme, which is based on Vehicle Ad Hoc Networks (VANET), for large parking lots through vehicular communication. The proposed systems could provide the drivers with real-time parking navigation services in large parking lots, intelligent antitheft protection, and friendly parking information dissemination.

Recently, the parking guidance system has been developed based on mobile phone and/or PDA to disseminate information. Yan et al. [20] proposed a novel, secure and intelligent parking system, which was called SmartParking, based on secured wireless networks and sensor communication. Wang and $\mathrm{He}$ [21] presented the design and implementation a prototype of Reservation-based Smart Parking System (RSPS) not only to broadcast real-time parking information to the drivers as part of a communal application on mobile device, but also to provide reservation service as part of user-targeted service.

\section{SYSTEM ARCHITECTURE}

In this section, the architecture and infrastructure of proposed smart parking service is presented.

\section{A. Overview of architecture}

The figure 1 shows the architecture of smart parking services in this paper.

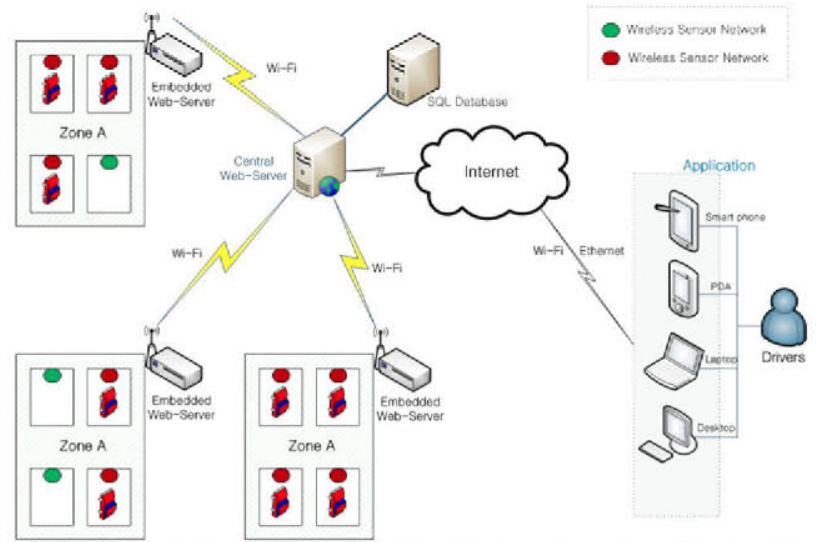

Fig. 1. The architecture of Smart Parking Systems based on Wireless Sensor Networks

The infrastructure of smart parking services consists of Wireless Sensor Networks (WSNs), Embedded Web-Server
(EWS), Central Web-Server (CWS), and Mobile Phone Application (MPA) of vehicle driver such as smart phone and PDA (Personal Digital Assistant) as well as laptop and desktop computer. In this smart parking service, the main objectives are the following:

- Wireless sensor networks module

- Collect sensor data

- Check parking slot state in real-time

- Send parking slot information to embedded webserver

- Embedded Web-Server

- Receive parking information from wireless sensor networks

- Send them with the position of parking zone to central web-server

- Central Web-Server

- Receive parking slot information from embedded web-server

- Display the parking slots state of parking zone in real-time

- Send these information to mobile phone application

- Save these information in SQL database

- Mobile device of driver

- Connect to central web-server

- Receive parking slot information from central web-server

- Display the real-time monitoring of parking slots state in the nearest parking zone

\section{B. Wireless Sensor Networks}

Wireless sensor network modules detect a parking slot state to read the measured result of the light sensor in real-time, and then to send this information to embedded web-server. Figure 2 shows the hardware architecture of wireless sensor networks nodes on the right and an actual photograph on the left. This wireless sensor networks node is a micro device equipped with

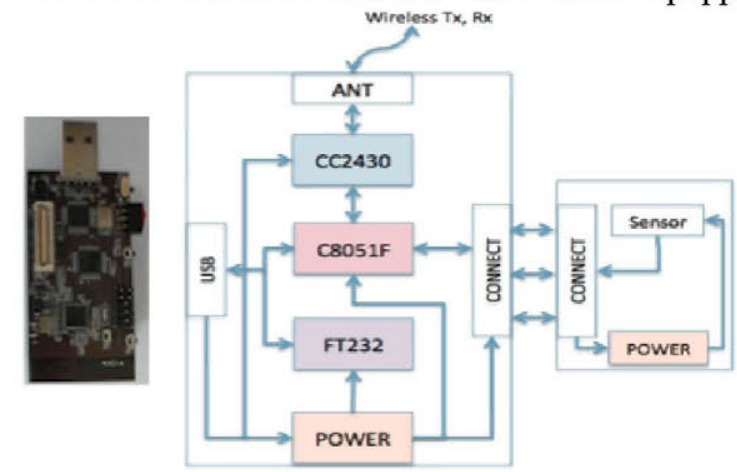

Fig. 2. The hardware architecture of Wireless Sensor Networks: (Left) actual photograph (Right) hardware architecture

a Intel Enhanced 8051 core, which is an 8-bit MCU working with a $32 \mathrm{MHz}$ system clock and has $128 \mathrm{Kbytes}$ in-system programmable flash memory, 8Kbytes RAM on the chip, a wireless communication module $\mathrm{CC} 2430$, which is a low power $2.4 \mathrm{GHz}$ IEEE 802.15 .4 compliant RF transceiver, a 
FTDI USB device as FT232, and a sensor board with light, temperature, humidity and a microphone.

The software of wireless sensor network node is based on Tiny OS [22] that is the most widely used operating system in wireless sensor networks. The software architecture consists of two layers, application layer and systems layer, as it is shown in Figure 3. The application layer has some modules such as detection, data collection, routing, and time synchronization to provide necessary services for parking place monitoring. The system layer contains some supporting modules for applications, such as ADC (Analog to Digital Converter) control, MAC (Media Access Control) control, Serial or USB interface debugger, data dispatching and forwarding, data aggregation, sensor measurement, and local debugger. The significance of extracting some functions of a system layer is that these can be ported to other applications easily.

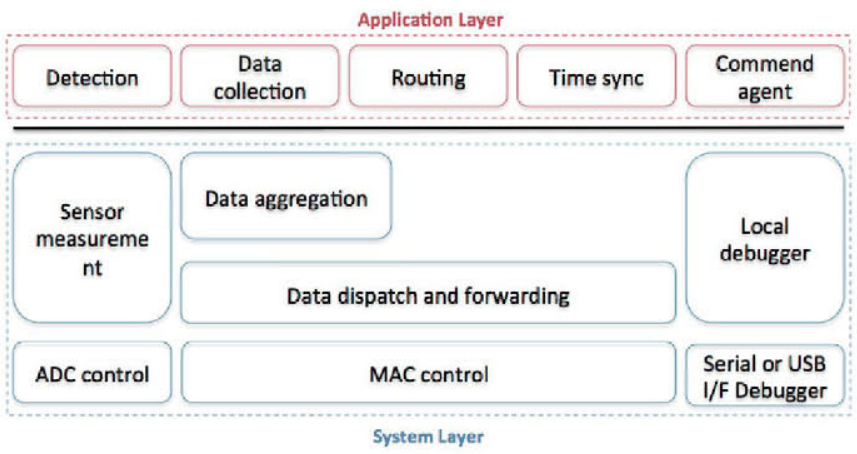

Fig. 3. The software architecture of two layers as application layer and systems layer

\section{Embedded Web-Server}

Embedded web-server receives the parking slot information from wireless sensor networks, collects these information, and displays the parking slots state in real-time. The parking slots are indicated on web-page within embedded web-server of parking zone. Parking information such as total parking slots number, available parking slots number, and parking zone position are transferred into central web-server.

In this research, embedded web-server is built on Arduino Uno, which is an open-source electronics prototyping platform

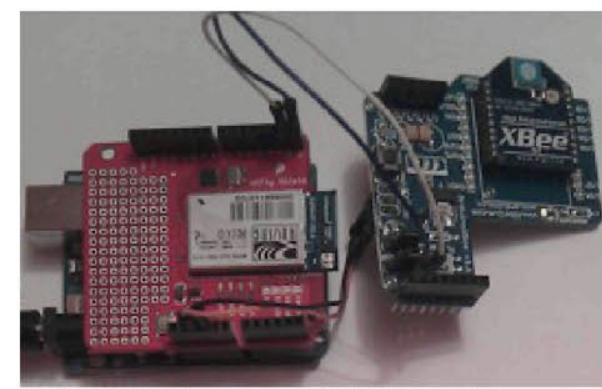

Fig. 4. The embedded web-server with Wi-Fi module and wireless sensor node

based on flexible, easy-to-use hardware and software, with RN-131 Wi-Fi module as ultra-low power embedded TCP/IP Fig. 6. Mobile Phone Application based on HTML5 and JQuery mobile stack and ZigBee module, which uses the IEEE 802.15.4 platform using Google Maps API on Android Smart Phone.

networking protocol for fast point-to-multipoint or peer-to-peer networking with low-power.

\section{Central web-server}

Central web-server receives the parking information from embedded web-servers of parking zone, displays the parking slots state, and updates MySQL database, automatically.

In this paper, the central web-server is built on Linux Mint (Version 12) $\mathrm{PC}$ running Apache Tomcat 5 and MySQL database. And the central web-server updates the MySQL database as it receives fresh reports from the network. The user-interface of web application is implemented based on HTML5 with JQuery mobile platform as shown in figure 5. Figure 5 shows the map of the parking zone and which parking spaces are vacant or occupied. These web pages can be connected using any web browser.
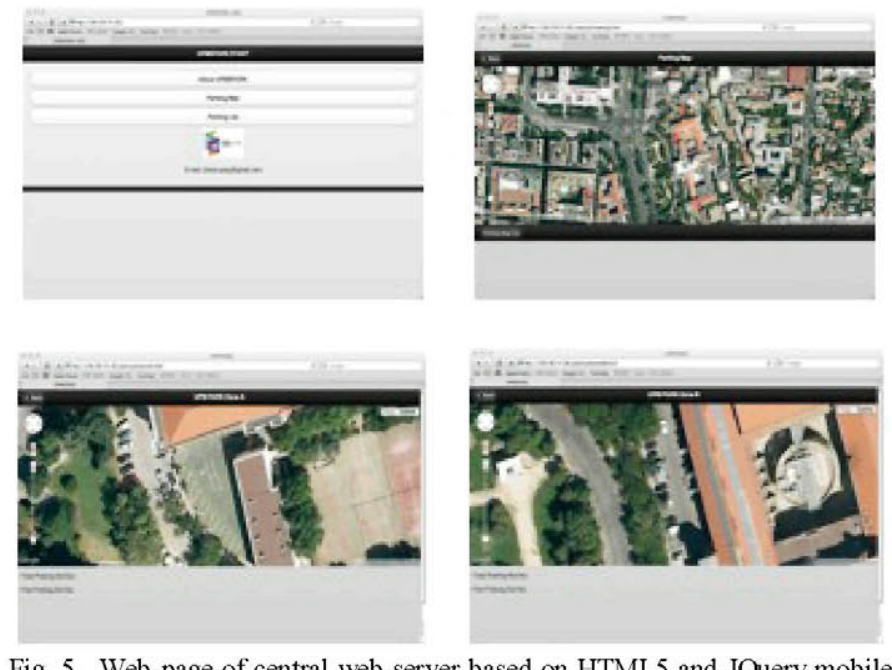

Fig. 5. Web-page of central web-server based on HTML5 and JQuery mobile platform using Google Maps.
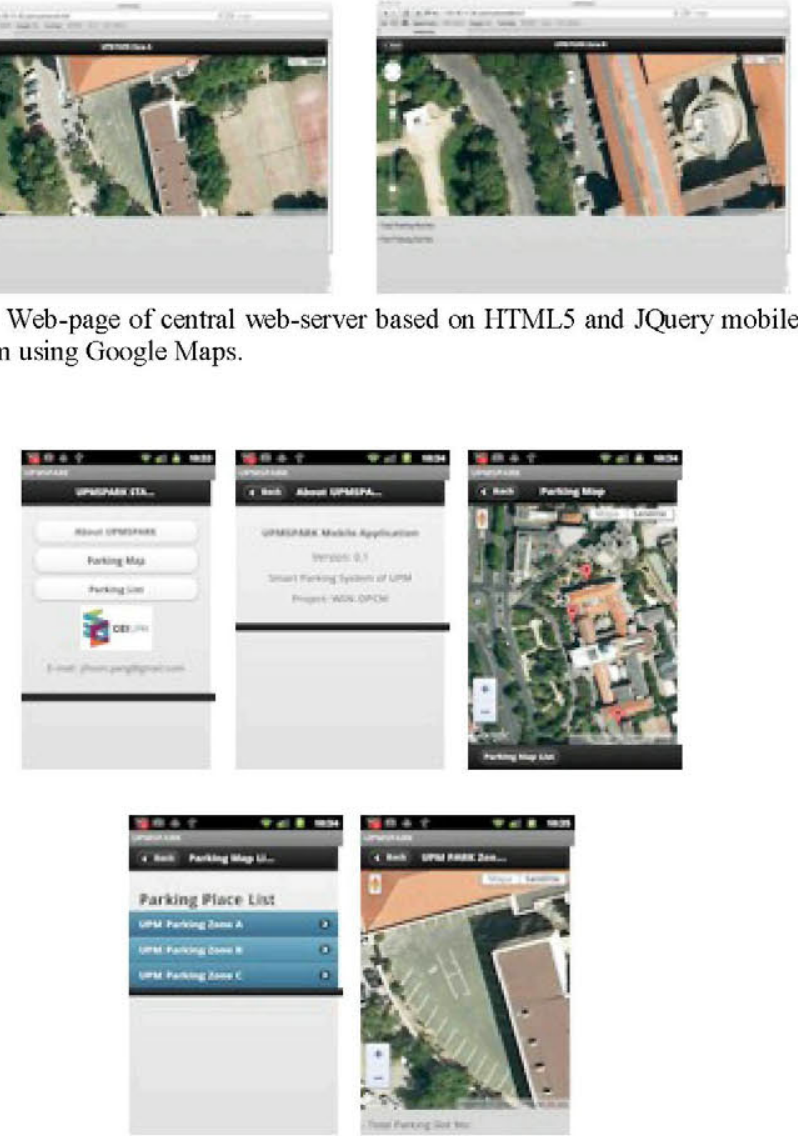

(n)

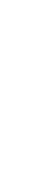




\section{E. Mobile Phone Application}

Mobile phone application is based on Android and it is shown in figure 6 . The mobile phone is used to access Internet, over Wi-Fi or a $3 \mathrm{G}$ cellular network, to obtain the information of parking availability and parking zone position from the central web-server. The Android phone application is developed in JAVA based on Android SDK.

Besides, these web pages are implemented on HTML5 with JQuery mobile platform in order to extend other mobile device as iPhone. For example, figure 7 shows same web user interface like android phone's web-pages (figure 6) using iPhone simulator.
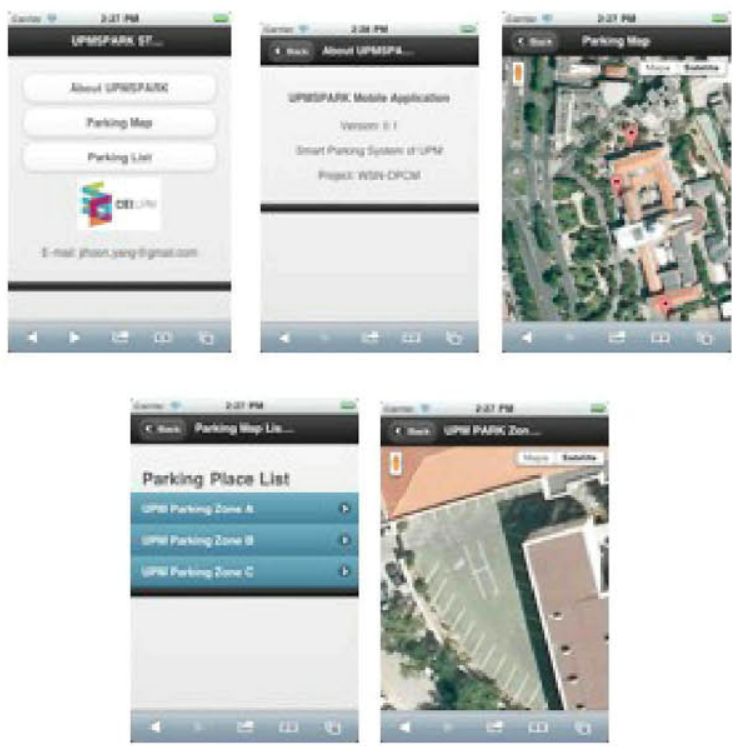

Fig. 7. iPhone user-interface (using iPhone simulator) based on HTML5 and JQuery mobile platform using Google Maps API like android user-interface as figure 6.

\section{PROTOTYPE IMPLEMENTATION}

In this section, we explain the prototype implementation of smart parking service for monitoring parking slots state. The prototype system is developed as a proof of concept to meet the real-time requirements of parking monitoring services. This section describes the development environment, service scenario and monitoring experiments.

\section{A. Development Enviroment}

To implement the smart parking system, we use four wireless sensor network modules with light sensor. We have modeled this prototype for four parking slots in our initial experiments. The parking zone has been deployed from Universidad Politecnica de Madrid using GoogleMaps. The mobile phone application is developed by using Android smart phone as HTC Nexus one model (android version 2.3).

\section{B. Service Scenario}

Figure 8 illustrates the flowchart of service scenario for smart parking services with respect to wireless sensor networks, embedded web-server, central web-server and mobile phone application in this paper. The process of this prototype system for monitoring service consists of the following four steps:

- Step 1 (WSN): Collect sensor data and check parking slot state such as vacant slot or occupied slot.

- Step 2 (EWS): Receive parking information, display them on web-page, and send this information.

- Step 3 (CWS): Display parking slots state on web-page, save them in database, provide parking information to mobile phone application.

- $\quad$ Step 4 (MPA): Connect to central web-server, monitor parking slots state in real-time.

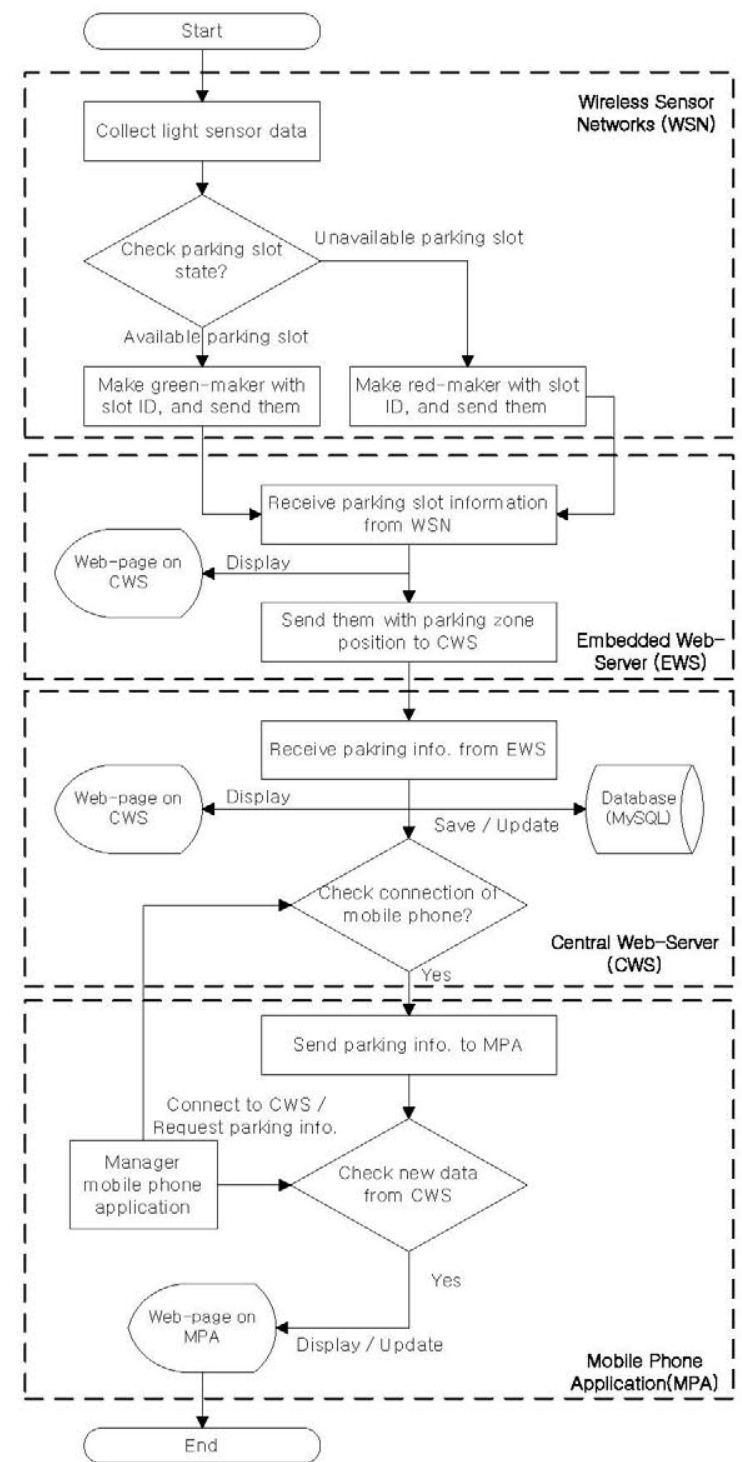

Fig. 8. Flowchart of service scenario for smart parking service with respect to wireless sensor networks, embedded web-server, central web-server and mobile phone application

And also, we present four scenarios as following: all parking slots are vacant, all parking slots are occupied, two vehicle are parked, and three vehicle are parked. 


\section{Monoritoring Experiements}

In these experiments, the red-marker on GoogleMaps indicates as an occupied parking slot, and a vacant parking slot is illustrated by the green-marker.

Figure $9 \sim 12$ show parking slots state on embedded webserver, central web-server and mobile phone application (Android HTC Nexus-One smart phone) in real-time. If

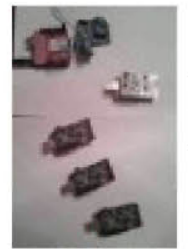

(a) WSN

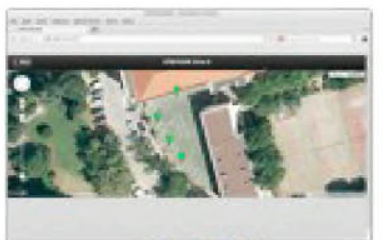

(c) CWS

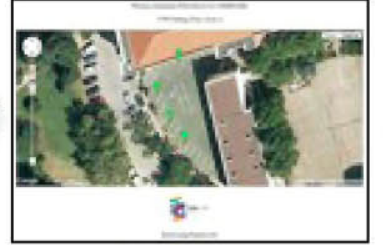

(b) EWS (d) MPA

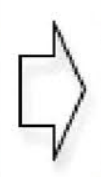

Fig. 9. The state of parking slots (Full free parking slots): (a) wireless sensor networks, (b) embedded web-server, (c) central web-server, (d) mobile phone application.

wireless sensor network node has a cover, the parking slot become unavailable for parking, then red-marker is indicated on web-page of embedded web-server, central web-server. Therefore, the vehicle driver will be able to confirm the parking slots state using mobile phone application in real-time. On the other hand, if wireless sensor network node does not the cover, the parking slot become available for parking, then the green-marker is indicated.

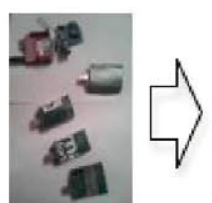

(a) WSN

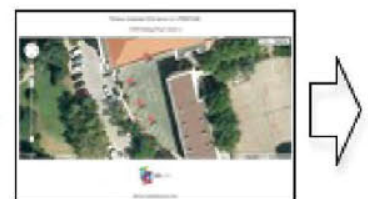

(b) EWS
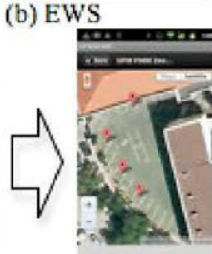

(d) MPA
Fig. 10. The state of parking slots (Full no free parking slots): (a) wireless sensor networks, (b) embedded web-server, (c) central web-server, (d) mobile phone application.

\section{CONCLUSIONS}

This paper proposes a set of smart parking services based on Wireless Sensor Networks. We design and implement a prototype system that allows vehicle drivers to effectively find the vacant parking spaces. The proposed smart parking system consists of wireless sensor networks, embedded web-server, central web-server and mobile phone application as Android and iPhone. We also implemented a prototype system, which

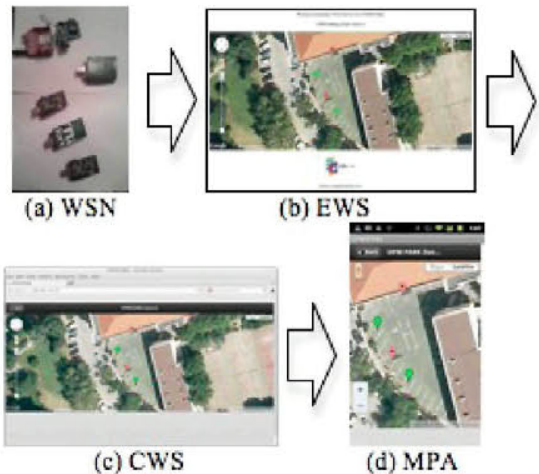

Fig. 11. The state of parking slots (two free parking slots and two no free parking slots): (a) wireless sensor networks, (b) embedded web-server, (c) central web-server, (d) mobile phone application.

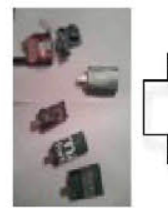

(a) WSN

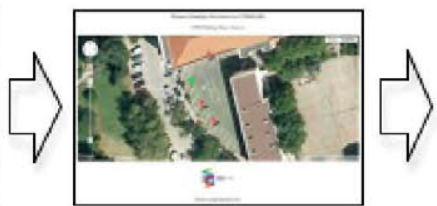

(b) EWS

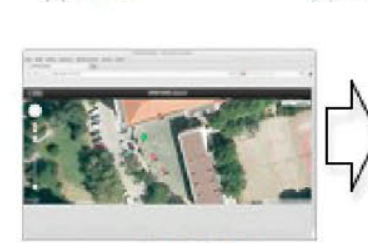

(c) CWS

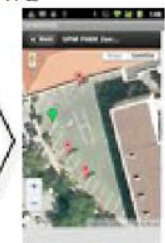

(d) MPA
Fig. 12. The state of parking slots (one free parking slot and three no free parking slots): (a) wireless sensor networks, (b) embedded web-server, (c) central web-server, (d) mobile phone application.

can effectively satisfy the requirements of WSN-based parking slots monitoring service. In this system, low-cost wireless sensors networks module are deployed into each parking slot equipped with one sensor node. The state of the parking slot is detected by sensor node and is reported periodically to the embedded web-server via the deployed wireless sensor networks. And this information is sent to central web-server using Wi-Fi networks in real-time. Besides, the vehicle driver can find vacant parking lots using their mobile devices. Though this prototype system we demonstrate that the proposed architecture can effectively satisfy the requirements of smart parking service and we believe that wireless sensor networks and mobile device application can be an encouraging technology to solve future parking management.

\section{ACKNOWLEDGMENT}

This research is partially funded by the ARTEMIS JU and the Spanish Ministerio de Industria, under the WSN-DPCM project. It is also co-funded by the BEAM project of Erasmus Mundus action. 


\section{REFERENCES}

[1] I. F. Akyildiz, W. Su, Y. Sankarasubramaniam, E. Cayirci, "Wireless sensor networks: a survey," Computer Networks, vol. 38. Pp. $393-422$, 2002

[2] N. Xu. "A Survey of Sensor Network Application," abailable: $\mathrm{http}: / /$ enl.usc.edu/ $\sim$ ningxu/papers/survey.pdf

[3] D. Puccinelli and M. Haenggi, "Wireless Sensor Networks: Applications and Challenges of Ubiquitous Sensing," IEEE Circuit and Systems Magazine, pp.19-29, 2005.

[4] J. Valverde, V. Rosello, G.Mujica, J. Portilla, A. Uriarte, and T. Riesgo, "Wireless Sensor Network for Environmental Monitoring: Application in a Coffee Factory," International Journal of Distributed Sensor Networks, vol. 2012, Article ID 638067, 18 pages 2012.

[5] Tian He, et al., "Energy-Efficient Surveillance System Using Wireless Sensor Networks," Proceedings of the $2^{\text {nd }}$ international conference on Mobile systems, applications, and services, Boston, Massachusetts, USA June $6-9$, pp. $270-283,2004$.

[6] A. M. Tabar, A. Keshavarz, and H. Aghajan, "Smart home care network using sensor fusion and distributed vision-based reasoning," Proceedings of the $4^{\text {th }}$ ACM international workshop on Video surveillance and sensor networks, Santa Barbara, Califonia, USA, October 27, pp. 145 - 154 2006.

[7] A. Baggio, "Wireless sensor networks in precision agriculture", in $A C M$ Workshop on Real-World Wireless Sensor Networks (REALWSN 2005), Stockholm, Sweden, June 20-21, 2005

[8] M. Srivastava, R. Muntz, and M. Potkonjak, "Smart kindergarten: Sensor-based wireless networks for smart developmental problem-solving envi- roments," in Proceedings of the 7th Annual International Conference on Mobile Computing and Networking (MobiCom'01), Rome, Italy, pp. $132-138,2001$

[9] T. Fulford-Jones, D. Malan, M. Welsh, and S. Moulton, "CodeBlue: An ad hoc sensor network infrastructure for emergency medical care," in International Workshop on Wearable and Implantable Body Sensor Networks, London, UK, 2004.
[10] P. White, "No Vacancy: Park Slopes Parking Problem And How to Fix It," http://www.transalt.org/newsroom/releases/ 126

[11] N. True, "Vacant parking space detection in static images." University of California, San Diego, 2007

[12] Q. Wu, C. Huang, S. Y. Wang, W. C. Chiu, and T. Chen, "Robust Parking Space Detection Considering Inter-Space Correlation," IEEE international Conference on Multimedia and Expo, Beijing, China, July 2 -5 , pp. 659-662, 2007.

[13] Siemens AG. Intelligent Traffic Systems. http://www.siemens.com/traffic

[14] V. W. S. Tang, Y. Zheng, and J. Cao, "An Intelligent Car Park Management System based on Wireless Sensor Networks," Proceedings of the 1st International Symposium on Pervasive Computing and Applications, Urumchi, Xinjiang, China, August, pp. 65 - 70, 2006.

[15] Crossbow Technologies, http://www.xbow.com

[16] M. Caliskan, D. Graupner, and M. Mauve, "Decentralized discovery of free parking places," In VANET '06: Proceedings of the 3rd international workshop on Vehicular ad hoc networks, New Tork, NY, USA, pp. 30 29,2006

[17] J. P. Benson, T. O'Donovan, P. O'sullivan, U. Roedig, and C. Sreenan, "Car-Park management using wireless sensor networs," in Proceedings of $31^{\text {st }}$ IEEE conf. Local Computer Networks, Tampa, pp. 588-595. 2006.

[18] V. K. Boda, A. Nasipuri, and I. Howitt, "Design Considerations for a Wireless Sensor Network for Locating Parking Spaces," Proceedings of IEEE SoutheastCon, Richmond, VA, March, pp. 698-703, 2007.

[19] R. Lu, X. Lin, H. Zhu and X. Shen, "SPARK: A New VANET-based Smart Parking Scheme for Large Parking Lots," in Proceedings of IEEE INFOCOM'07, 2007.

[20] G. Yan, W. Yang, D. B. Rawat and S. Olariu, "SmartParking: A Secure and Intelligent Parking System," IEEE Intelligent Transportation Systems Magazine, vol. 3(1), pp. $18-30,2011$.

[21] Hongwei Wang and Wenbo He, "A Reservation-based Smart Parking System," The First International Workshop on Cyber-Physical Networking Systems, pp. 701 - 706, 2011.

[22] Tiny OS, http://www.tinyos.net 